\title{
Book Review: Flow Measurement Handbook (Second Edition) Roger C. Baker
}

The second edition of this magisterial work was published towards the end of 2016; the first edition, already a familiar reference to many readers here, was released in 2000 and reviewed in FMI by Richard Furness [1]. We are thus offered a rare opportunity to apply the principle of crosscorrelation (ably described in section 14.5 of the new edition) to the state of the flow metering art itself: two very detailed snap-shots are provided at a fixed distance apart (in time), as recorded by a reliable observer (the estimable Professor Baker). From these snap-shots we may attempt to deduce the rate of change of the technology, and perhaps determine which features are developing, maintaining, or losing their importance over time.

The target audience for the Flow Measurement Handbook is primarily those in industry who have any dealings with flowmeters, from engineers to marketeers, though the readership of the first edition has extended well into academia too. The emphasis is on mechanical and fluid engineering; descriptions of the electrical engineering and signal processing aspects of flow meter design are sufficient to provide a basic understanding of meter function, but Professor Baker does not attempt to provide a definitive account of these rapidly evolving fields. Given the broad readership, each topic under consideration is explained from first principles through to the state of the art, drawing on both published research and the marketing claims of manufacturers. References to relevant industrial standards are given, but the author repeatedly and rightly advises the reader to consult these standards directly, rather than relying on such summaries as are provided in the text. Sufficient mathematics is embedded in the main text in order to explain the principles of each measurement technique, while more detailed analysis, where required, is provided in one or more appendices at the end of the chapter.

The structure of the second edition broadly follows that of the first. Introductory chapters are provided on Fluid Mechanics Essentials; Specification, Selection and Audit; and Calibration. The second edition augments Chapter 2 on Fluid Mechanics Essentials (which summarises the basics of 
flow in a circular pipe, unsteady flow, pulsation, compressible flow and various forms of multiphase flow) with an Appendix. This covering flow profile equations and flow conditioning, and further provides a brief introduction to CFD as applied to flow metering, including useful references from the literature. Chapter 3 on Specification, Selection and Audit is little changed; it offers a guide on drawing up a flow measurement specification for any particular application, and mapping that specification onto a potential solution using a selected flow technology and supplier, taking into account the full range of issues from the technical through to the commercial. Chapter 4 provides a thorough introduction to the business of Calibration, primarily by explaining the variety of laboratory (e.g. gravimetric, sonic nozzle) and field (e.g. prover) techniques used for pure liquids and gases. A new Appendix provides an overview of recent calibration developments described in the literature (e.g. for multiphase flow), and gives two brief case studies of actual calibration facilities.

The bulk of the book consists of 17 chapters, each devoted to a specific flow metering technology. Three of these describe new technologies not covered in the first edition: Magnetic Resonant Flowmeters, Acoustic and Sonar Flowmeters, and Multiphase Flowmeters. Each chapter has a broadly similar structure, adapted to explain the most significant issues in successfully applying the metering technology in question. The operating principle is introduced, using the minimum mathematical description of both the physics and the signal processing needed to obtain flow measurement information. The key issues for the technology in question are then discussed, covering such factors as design, installation, measurement performance and calibration, reflecting the state of the art in current industrial practice. Most chapters have a useful Application, Advantages, Disadvantages section which summarises the pros and cons of the particular flow metering technology. Appendices, often quite lengthy, provide more detailed mathematical background and/or (in the second edition) an update on the most recent developments.

Chapter 5 describes Orifice Plate Meters, and includes overviews of device-specific issues such as the influence of flow pulsation, high pressure plate deflection, and multiple flow 
components. The revised Appendix 5A gives an account of the various Orifice Discharge Coefficient equations as adopted by international standards, while the new Appendix $5 \mathrm{~B}$ reviews recent developments in the literature, covering such topics as installation effects, contamination, drain holes, flow conditioning and CFD modelling.

Chapter 6 describes Venturi Meters and Standard Nozzles, Chapter 7 covers Critical Nozzles, while Chapter 8, titled Other Momentum-Sensing Meters, includes a wide range of related proprietary meters such as the variable area, V-cone and wedge meters. Chapters 6 and 7 include new appendices providing overviews of recent research.

Chapter 9 looks at the range of positive displacement meters for liquids, gases, and multiphase flows. Chapter 10 covers turbine and related flowmeters, including an additional section on dual rotor meters. Both Chapters have new Appendices describing recent developments in theoretical and experimental research.

Chapter 11 describes vortex meters, and includes a section on the related swirl meters. In this Chapter, new developments in the technology are integrated into the main body of the text, particularly under section 11.3.4 Sensing Options, which includes an overview of recent approaches to vortex meter signal processing.

Chapter 12 looks at electromagnetic flowmeters, where recent advances are described in both an additional section 12.12 New Industrial Developments, and an extended Appendix covering verification, non-conducting dielectric fluids, and the metering of liquid metals in nuclear power applications. This is followed by Chapter 13, the first entirely new Chapter in the second edition, which introduces the emerging flowmeter technology based on (nuclear) magnetic resonance.

Chapter 14, on the Ultrasonic flowmeter (along with the Doppler and Cross-Correlation meters), has benefitted from substantial redrafting and expansion, with an additional section on clamp-on metering, coverage of new sensing and signal processing techniques, and a significantly 
enlarged discussion of installation effects. A new section in the Appendix covers recent developments in the modelling of ultrasonic meters. Chapter 15, on Acoustic and Sonar Flowmeters, is another newly added Chapter, introducing the SONARtrac and Expro clamp-on sonar-based meters.

Chapter 16 is titled "Mass Flow Measurement using Multiple Sensors for Single-Phase Flow", which covers some interesting but not widely applied techniques such as a hydraulic Wheatstone bridge system. Material originally from the second half of this chapter has been significantly expanded to create an additional Chapter (17) in the second edition covering Multiphase Flowmeters, reflecting the substantial developments in this technology over the last decade and a half. The text provides a useful overview of the various multi-sensor configurations applied to metering multiphase flow, with an extensive range of recent references for the interested reader.

Chapters 18 covers Thermal Mass Flowmeters for both liquids and gases, while Chapter 19 documents another relatively obscure technology, Angular Momentum Devices, which use mechanical rotation to determine liquid mass flow, an approach largely superseded by Coriolis mass flow metering, which is covered in Chapter 20. This, like the Chapter on ultrasonic flow-metering, has been substantially reworked in the new edition to provide an up-to-date and integrated account of a rapidly evolving technology. Key developments described include flowtube modelling, the new digital electronics and signal processing, and the extension of Coriolis mass flow metering into twophase and three-phase applications.

Chapter 21, on Probes for Local Velocity Measurement in Liquids and Gases, is the last to describe a specific flow metering technology, and covers Pitot tubes and a variety of insertion probes. Note that hot wire and laser Doppler anemometry are not included here, as they are considered to be primarily laboratory rather than industrial techniques. Other flow metering topics not covered in the handbook include metering pumps, flow controllers, the measurement of solids and granular materials, and open channel flow measurement. 
The final section of the book considers broader issues, and shows the most significant reworking from the previous edition, reflecting perhaps the rapidly changing industrial environment in which flow-metering is required to operate.

Chapter 22 discusses a new topic of Verification and In Situ Methods for Checking Calibration, which is becoming an increasingly important concern for industrial measurement. How do we know our flow measurements are valid? When should recalibration take place? Two approaches are considered, "verification" i.e. the generation of diagnostic information within the meters themselves, and the generation of independent flow estimates via the network of installed plant pipework itself (e.g. by monitoring pressure losses) or by applying clamp-on meters.

Chapter 23 on Remote Data Access Systems provides a basic overview of the various standard means by which flow measurement information and other data are communicated from a flowmeter to other system components in industrial applications. This is an area that has seen significant change over the last 15 years, whereby the old idiosyncrasies of industrial communications have been subsumed into the universally applied techniques of the IT sector (e.g. Ethernet), and this change is reflected in a thorough redrafting and simplification of the text.

Chapter 24, Final Considerations, ponders the future of flow metering, with an emphasis on the importance of manufacturing quality in delivering accurate measurement performance, but looking in particular to micro-machined transducers, signal processing, and verification techniques to provide the next significant breakthroughs.

Professor Baker's second edition is a thoroughly welcome volume, and highly recommended to new readers. As for those old enough to have a copy of the first edition on their shelves, it is likely that the ninety pages of updated and judiciously selected references would on their own justify the investment. More broadly, however, and returning to the observations that opened this review, 
perusing the second edition avails the opportunity to see, through the eyes of a trusted guide, where and how our craft has evolved since the turn of the century.

References

[1] R. Furness, Review of Flow Measurement Handbook, Flow Measurement and Instrumentation 12 (2001) 233-234. 